\title{
ORIGINAL ARTICLE \\ Medical complications during pregnancy and childbirth in women with SCI in Switzerland
}

\author{
S Bertschy ${ }^{1,2}$, C Bostan ${ }^{1,2}$, T Meyer $^{3}$ and J Pannek ${ }^{4}$
}

Study design: A retrospective interview study of mothers with spinal cord injuries (SCIs) who gave birth over the last 15 years. Objectives: To identify the medical complications of women with SCls during pregnancy and childbirth in Switzerland and to describe how they dealt with these complications.

Settings: Swiss Paraplegic Research in Nottwil, the University of Lausanne and participants' homes.

Methods: Data were collected by self-reported questionnaires and descriptive analysis was conducted.

Results: Seventeen women with SCls who gave birth to 23 children were included. Thirteen of the women were paraplegics and four were tetraplegics. All of them practiced an independent bladder management. Three women changed their bladder management techniques during pregnancy. Five women reported an increased bladder evacuation frequency during pregnancy, and six women reported a new onset or increase in incontinence. We observed no significant increase in bowel dysfunction or skin breakdown due to their pregnancies. Ten women were hospitalised during the course of their pregnancies. Aside from urinary tract infections/ pyelonephritis, women were hospitalised for falls, hypertension, pneumonia, preeclampsia, pre-term labour or tachycardia.

Conclusion: The results of our study clearly demonstrated that, although medical complications are not infrequent during pregnancy in women with SCls, pregnancy and delivery in this group of women are possible without posing intolerable risks to the mothers or the children. Urological problems seemed to be the most frequent complication during pregnancy.

Spinal Cord (2016) 54, 183-187; doi:10.1038/sc.2015.205; published online 1 December 2015

\section{INTRODUCTION}

A traumatic spinal cord injury (SCI) does not impair fertility in women. Following a phase of amenorrhoea, women with SCIs may become pregnant. ${ }^{1}$ As researchers, we assert an increasing interest in this topic of women with SCIs.

Pregnancy, childbirth, parenting and motherhood in women with SCIs have all been researched from medical ${ }^{2-6}$ and psychosocial $^{7-10}$ perspectives. In addition, health-care providers' knowledge ${ }^{11}$ and accessibility as well as the availability of services ${ }^{12-15}$ have been assessed. However, different topics related to pregnancy and SCI care are still under-researched and therefore require more focused research. One of these topics is the potential medical complications that occur during pregnancy as addressed from the perspectives of the pregnant women.

Urological complications are of particular importance in pregnant women with SCIs. First, virtually all patients with SCIs suffer from neurogenic lower urinary tract dysfunction. ${ }^{16}$ An elevated storage pressure, either due to low bladder compliance or neurogenic detrusor overactivity, is a major risk factor for renal deterioration. ${ }^{17}$ Therefore, the primary objective of bladder management is to prevent renal damage. Furthermore, neurogenic lower urinary tract dysfunction may also lead to incontinence and recurrent symptomatic urinary tract infections (UTIs). In patients with SCIs, UTIs are the leading cause of septicaemia and are associated with a significantly increased mortality rate. ${ }^{18}$ In addition, both UTIs and incontinence significantly impair the quality of life of affected persons. ${ }^{19}$

Pregnancy is also known to have an impact on the lower urinary tract of neurologically intact women. The risk for bacteriuria for example is elevated in pregnant women. ${ }^{20}$ Thus, pregnant women with SCIs are likely at a higher risk for urological complications.

As a consequence, pregnant women with SCIs require specific paraplegiological and urological care. However, empirical evidence on urological complications in pregnant women with SCIs is scarce. A recent review demonstrated that there are virtually no data about the incidence, severity and management of urological complications in these patients. ${ }^{21}$ To get a clearer picture, we collected data from women who were concerned about their urological health and other specific SCI medical problems during their pregnancies. The objective of this study was to identify any medical complications and to describe how women with SCIs dealt with those complications during pregnancy and childbirth in Switzerland.

\section{MATERIALS AND METHODS}

\section{Study design}

A descriptive study was conducted that included mothers with traumatic or non-traumatic SCIs between 18 and 54 years of age with permanent residence in Switzerland who had given birth within the past 15 years and after their SCIs.

The study participants were recruited for a qualitative study. The aim of this qualitative study was to examine the health service experiences

${ }^{1}$ Swiss Paraplegic Research (SPF), Nottwil, Switzerland; ${ }^{2}$ Department of Health Sciences and Health Policy, University of Lucerne and SPF, Nottwil, Switzerland; ${ }^{3}$ Hannover Medical School, Institute for Epidemiology, Social Medicine and Health Systems Research, Hannover, Germany and ${ }^{4}$ Neuro-Urology, Swiss Paraplegic Center (SPZ), Nottwil, Switzerland Correspondence: S Bertschy, Swiss Paraplegic Research, Guido A, Zäch-Strasse 4, Nottwil 6207 Switzerland.

E-mail: sue.bertschy@paraplegie.ch

Received 29 April 2015; revised 13 October 2015; accepted 16 October 2015; published online 1 December 2015 
of women with SCIs during pregnancy and childbirth. ${ }^{22}$ The recruitment process was previously described..$^{22}$ In brief, women were recruited through the Swiss Spinal Cord Injury Cohor ${ }^{23}$ database and through snowball sampling in the disability community. ${ }^{24}$ Both lists were matched, and all retrieved names (94 names in total) were cross-checked. A total of 34 individuals were excluded based on language and age (54 years of age and older); in addition, seven names were doubles. A total of 60 women were invited to participate in the study. The response rate was $70 \%$ ( 42 women). These respondents were contacted by telephone for a second eligibility check. Of these mothers, 18 did not meet the eligibility criteria and seven declined to participate. Seventeen mothers expressed interest in taking part in the study, representing a final response rate of $28 \%$. This sample might not be considered a representative sample of pregnant women with SCIs in Switzerland, as there are no data on the total number of pregnant women and their distribution.

\section{Data collection}

After the qualitative interview sessions, participating women were asked to fill out a questionnaire and to retrospectively report on the most frequent secondary medical problems that resulted from their SCIs and that had developed during their pregnancies. The list was developed by considering the current literature and by involving health providers who had cared for pregnant women with SCIs. ${ }^{25}$ The questionnaire specifically asked about skin problems, bowel function, UTI frequency, mode of delivery, decubital ulcers, hospital admissions, alterations in medication, respiratory tract problems and changes in neurogenic lower urinary tract dysfunction symptoms.

In addition, patients were asked whether they took prophylactic measures against UTIs, decubitus and deep vein thrombosis, as well as what those measures were. As we were also interested in information related to women's bladder voiding techniques, we collected data from qualitative interviews. A recent study showed that voiding technique has a major influence on the incidence rates of UTIs. ${ }^{26}$

\section{Data analysis}

Descriptive statistics were used to analyse the data.

\section{RESULTS}

\section{Demographic data}

The demographic characteristics of the 17 participants are presented in Table 1. Thirteen women were paraplegic and four were tetraplegic. Although the mean age was 21 at the time of the injury, the mean age at the time of giving birth was 33 . The 17 women gave birth to 23 children altogether after their SCIs, and two women each had one child before their SCIs. The mean interval between SCI incidences and the birth of a first child was 12 years. At the time of motherhood, all mothers were either married or had stable relationships, and all women had health insurance.

\section{Specific changes in health management and medical complications during pregnancy due to SCIs}

Table 2 shows the health complications during pregnancy in women with SCIs, their treatments and the prevention measures taken.

No women reported skin problems. Two women paid special attention to the prevention of decubital ulcers (by resting more). Ten women performed prophylactic measures against deep vein thrombosis where nine of them used compressive stockings. No incidences of deep vein thrombosis were diagnosed during pregnancy.

Table 3 shows the bladder/bowel dysfunctions and medication changes that occurred in women with SCIs during pregnancy. Constipation was the most frequently reported complaint concerning bowel dysfunction, whereas diarrhoea, flatulence and abdominal gas occurred rarely. In summary, pregnancy did not significantly increase bowel dysfunction. Concerning drug treatments, three women either stopped taking their pain medications (such as Citalopram or
Tegretol) or stopped taking their anticholinergic treatment for detrusor overactivity. Two women reported that they stopped taking all their medication.

Regarding bladder management, ten women reported the use of intermittent self-catheterisation, four had an anterior root ('Brindley') stimulator and three emptied their bladder using the Credé method.

Three women changed their bladder management techniques during pregnancy. One used an indwelling catheter prescribed by her gynaecologist, one reduced her anticholinergic treatment for detrusor overactivity and one did not perform onabotulinumtoxin injections in the detrusor at the scheduled times.

Five women reported an increased bladder evacuation frequency during pregnancy, six women reported the onset of incontinence or an increase in incontinence. As a consequence, one woman took the initiative to use larger incontinence devices (diapers). Although nine women experienced no change in UTI frequency, six women reported an increased number of UTIs. None of the women using a stimulator reported an increase in UTI frequency during pregnancy. Although UTIs were most frequently treated by antibiotics $(n=7)$, four women did use some combination of homoeopathy, UTI prophylaxis and phytotherapy (bearberry-leaf tea and cranberry extract) (Table 3).

Ten women were hospitalised during the course of their pregnancies. In four women, UTIs/pyelonephritis led to hospitalisation, making it the most common reason for hospitalisation during pregnancy. Besides UTI/pyelonephritis, women were hospitalised for falls, hypertension, pneumonia, preeclampsia, pre-term labour or tachycardia.

\section{Obstetrical issues}

One woman was interviewed during (the last trimenon of) pregnancy, and sixteen of the seventeen women gave birth to at least one child. In 15 women, delivery took place before the calculated date of birth (from 1 to 5 weeks early). Five women had vaginal births, whereas eleven women underwent caesarean sections (Table 4). Moreover, whereas all women received either general (8 women) or epidural ( 3 women) anaesthesia for their caesarean sections, only one woman who gave vaginal birth required general anaesthesia. This woman also delivered her child on the scheduled day.

\section{DISCUSSION}

We described the medical complications that occurred during the course of pregnancy in women with SCIs as retrospectively reported

\section{Table 1 Demographics of participants}

Mean (range)

Age, years

At time of injury

$21(2-37)$

At time of motherhood

$33(25-40)$

Time SCl-motherhood

12 (2-23)

Education

$n$

Apprenticeship

5

Bachelor degree

Master degree

Postgraduate degree

Type of injury

Complete paraplegia

Incomplete paraplegia

Complete tetraplegia

Incomplete tetraplegia

4

6

2

$n$

7

6

1

3

Abbreviation: $\mathrm{SCl}$, spinal cord injury. 
Table 2 Health complications during pregnancy in woman with $\mathrm{SCl}$, treatments and prevention measures

\begin{tabular}{lclllr}
\hline Type of complication & $N$ & Treatment & $N$ & Prevention measure & $N$ \\
\hline Skin (pressure sores) & 0 & & Increased rest & Leg elevation \\
Thrombosis & 0 & & Medication (anticoagulants) & 2 \\
& & & Compressive stockings & 9 \\
& & & Taking shower & 4 \\
& & & Lymph drainage & 1 \\
Urinary tract infections & 11 & Antibiotics & 8 & & \\
& & Cranberries & 1 & & \\
& & Homoeopathy & 1 & \\
Respiration problems & 1 & & & Laxative tea & \\
Bowel problems & 10 & & &
\end{tabular}

Abbreviation: $\mathrm{SCl}$, spinal cord injury.

aultiple answers.

Table 3 Bladder/bowel dysfunction and medication changes during pregnancy in woman with $\mathrm{SCl}$

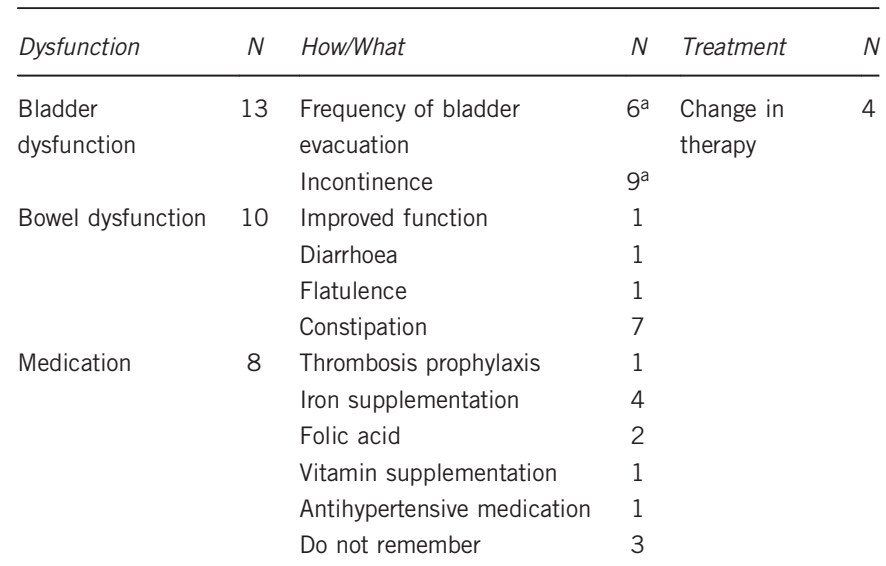

aultiple answers.

by the women. In particular, we assessed skin, bladder, bowel and respiratory problems, as well as the prophylactic and treatment measures used by the pregnant women. In addition, we identified the women's experiences of any medical problems related to their deliveries.

According to women's reports, the majority of changes in medical procedures were related to bladder management, including changes in medication and changes in bladder evacuation methods. These changes led to an increased rate of UTIs in a substantial subset of women. It is well known that the use of indwelling catheters leads to an increased UTI rate. ${ }^{27}$ Most anticholinergic drugs should not be taken at least during the first trimenon of pregnancy because fetal malformations were observed in animal in experiments that used these drugs, ${ }^{28}$ and onabotulinum toxin is also not licensed for use during pregnancy. Given this, ineffective treatment of detrusor overactivity may occur, such as by decreasing the dosage of anticholinergic medication or by suspending onabotulinumtoxin injections in the detrusor. This usually leads to incontinence, which is frequently treated by inserting an indwelling catheter, especially if intermittent catheterisation becomes more difficult during the course of pregnancy. In addition, detrusor overactivity per se is linked to an increase in UTI frequency. ${ }^{29}$ Therefore, the need to alter treatments for detrusor overactivity during pregnancy may increase the risk of having UTIs. Patients with Brindley stimulators neither performed intermittent
Table 4 Delivery, birth type and anaesthesia indications for 16 out of 17 woman $^{\text {a }}$

\begin{tabular}{llc}
\hline Indicators & & $N$ \\
\hline $\begin{array}{l}\text { Week of delivery before the term } \\
\text { (weeks) }^{\text {b }} \text { mean (min, max) }\end{array}$ & & $2.4(1,5)$ \\
Type of birth & Caesarean section & 11 \\
& Vaginal birth & 5 \\
Anaesthesia & General anaesthesia & 4 \\
& Epidural anaesthesia & 8 \\
\hline
\end{tabular}

${ }^{a}$ One woman was still pregnant at the time of data collection.

bPre-term $n=14$.

catheterisation nor received treatment for detrusor overactivity. Given that the detrusor becomes areflexic after this procedure, ${ }^{30}$ it was not surprising that this group of women did not suffer from UTIs during pregnancy.

In four women, hospitalisation was required because of pyelonephritis. It is well known that pyelonephritis may lead to premature labour. As the overwhelming majority of the study participants (14/16) experienced premature labour in our study, we cannot reliably relate pyelonephritis to this phenomenon in our study.

The study results show that women used numerous prophylactic measures during pregnancy to avoid complications. No skin breakdown or thrombosis occurred during pregnancy. One possible reason for this enhanced bodily awareness could be the educational nature of the rehabilitation programs in the rehabilitation centres in Switzerland.

Given the high number of bowel problems in individuals with SCIs, ${ }^{31}$ we were surprised that none of the women asked their physicians for medical advice concerning these problems. We assume that this complication was not sufficiently important to the women to warrant seeking help. In addition, we think that these women may believe that pregnancy requires such forms of tolerance.

Several studies ${ }^{32-34}$ describe higher rates (ranging from 18 to $49 \%$ ) of caesarean sections among women with SCIs compared with able-bodied women, and it is unclear whether the predominant causes are gynaecological in origin. ${ }^{32,33}$ In an American study, ${ }^{34}$ the most common reasons for a caesarean section are 'elective repeat procedure, failure in progress in labour and pelvic instability.' In 2013 in Switzerland, every third birth occurred through a caesarean section, ${ }^{35}$ which is an average frequency compared with other European countries. Our results also show a high tendency towards caesarean sections (69\%) in women with SCIs. The reasons for this finding are unclear. A qualitative study examined the subjective health needs and services used by women with SCIs from Switzerland during pregnancy; ${ }^{22}$ it described that some women reported not having had a choice about their birthing process. The women thought that the medical providers may have been unfamiliar in dealing with patients with SCIs or else felt anxious about it. A medical chart review study could add more insight into this theme.

Women described several medical complications and how they dealt with. Remarkably, they did not describe or named the phenomenon of autonomic dysreflexia, which is a common and severe complication during labour in SCI patients. ${ }^{32}$ We would like to point out, however, that this study reports the subjective perspective of the women experiencing medical complications. By not mentioning some medical needs does not mean that they are not important or do not occur. In contrast, this may reflect the high competence of the care deliverer, taking risks into account without unsettling women during the birth 
process. As we report retrospective data, we assume that women remembered rather medical needs during pregnancy course than during birth. Despite the lack of autonomic dysreflexia in our SCI population, the reader should not assume that the danger does not exist. Practitioners managing labour in SCI women still need to be acutely aware of the possibility of autonomic dysreflexia and also be well-versed in treating potential paroxysmal cardiovascular instability.

In summary, our findings demonstrate that pregnancy in women with SCIs may lead to medical problems. Patients with SCIs frequently use medications on a permanent basis, such as for pain, spasticity or bladder management. Although these drugs may not be licensed for use during pregnancy, no major complications due to alterations in medication were reported. According to our data, bladder management during pregnancy seems to be a major issue, but it was manageable for all women without irreversible complications. It is noteworthy that many possible complications, such as deep vein thrombosis or pressure sores, did not occur. This may indicate that providing adequate education to patients in primary rehabilitation leads to low complication rates because of sufficient self-care, even in 'high risk' situations.

\section{CONCLUSIONS}

The results of our study clearly demonstrate that, although medical complications are not infrequent during pregnancy in women with SCIs, pregnancy and delivery in this group of women are possible without posing an intolerable risk to the mothers or the children. Urological problems seem to be the most frequent complications experienced during pregnancy.

\section{Study limitations}

The comparably small number of participants is a limitation of this study. No official register exists of women with SCI who gave birth in Switzerland. We had to rely on the SwiSCI cohort study and recruiting directly in the disability community with the help of our study participants. This variation allowed us to recruit all over the country, but still we do not have a firm ground to make statements regarding the representativeness of our sample.

Furthermore, the questionnaire specifically mentioned possible medical complications, whereas other possible complications were not mentioned. With an open question at the end of the questionnaire, the women had the possibility to mention further complications. In addition, the questionnaire was not validated, meaning we do not have data on the accuracy of these women's retrospective accounts with regard to medical complications or interventions during pregnancy. The complications indicated were typical of the most frequent medical problems reported during pregnancy. For us it was important to assess whether these problems did occur in the survey participants. Even more significantly, we wanted to determine how the women coped with these problems. In addition, any significant, far-reaching complications may have a higher likelihood of being remembered.

\section{DATA ARCHIVING}

There were no data to deposit.

\section{CONFLICT OF INTEREST}

The authors declare no conflict of interest.

\section{ACKNOWLEDGEMENTS}

This study has been financed in the framework of the Swiss Spinal Cord Injury Cohort Study (SwiSCI, www.swisci.ch), supported by the Swiss Paraplegic Foundation. The members of the SwiSCI Steering Committee are as follows:
Olivier Dériaz, Xavier Jordan (Clinique Romande de Réadaptation, Sion); Michael Baumberger, Hans Peter Gmünder (Swiss Paraplegic Center, Nottwil); Armin Curt, Martin Schubert (University Clinic Balgrist, Zürich); Margret Hund-Georgiadis, Kerstin Hug (REHAB Basel, Basel); Hans Georg Koch, Urs Styger (Swiss Paraplegic Association, Nottwil); Hardy Landolt (Representative of persons with SCI, Glarus); Hansjörg Koch (SUVA, Luzern); Mirjam Brach, Gerold Stucki (Swiss Paraplegic Research, Nottwil); and Martin Brinkhof, Christine Thyrian (SwiSCI Study Center at Swiss Paraplegic Research, Nottwil). This study has been financed in the framework of the Swiss Spinal Cord Injury Cohort Study (SwiSCI, www.swisci.ch), supported by the Swiss Paraplegic Foundation.

1 Reame NE. A prospective study of the menstrual cycle and spinal cord injury. Am J Phys Med Rehabil 1992; 71: 15-21.

2 Ghidini A, Simonson M. Pregnancy after spinal cord injury: a review of the literature. Top Spinal Cord Inj Rehabil 2011; 16: 93-103.

3 Smeltzer SC, Wetzel-Effinger L. Pregnancy in women with spinal cord injury. Top Spinal Cord Inj Rehabil 2009; 15: 29-42.

4 Sterling L, Keunen J, Wigdor E, Sermer M, Maxwell C. Pregnancy outcomes in women with spinal cord lesions. J Obstet Gynaecol Can 2013; 35: 39-43.

5 Signore C, Spong CY, Krotoski D, Shinowara NL, Blackwell SC. Pregnancy in women with physical disabilities. Obstet Gynecol 2011; 117: 935-947.

6 Krassioukov A, Warburton DE, Teasell R, Eng JJ. A systematic review of the management of autonomic dysreflexia after spinal cord injury. Arch Phys Med Rehabil 2009; 90: 682-695.

7 Tebbet M, Kennedy P. The experience of childbirth for women with spinal cord injuries: an interpretative phenomenology analysis study. Disabil Rehabil 2012; 34: 762-769.

8 Albright KJ, Duggan $\mathrm{CH}$, Rahman RO. Motherhood in the context of spinal cord injury. Top Spinal Cord Inj Rehabil 2009; 15: 43-58.

9 Alexander CJ, Hwang K, Sipski ML. Mothers with spinal cord injuries: impact on marital, family, and children's adjustment. Arch Phys Med Rehabil 2002; 83: 24-30.

10 Grue L, Lærum KT. 'Doing motherhood': some experiences of mothers with physical disabilities. Disabil Soc 2002; 17: 671-683.

11 Burch A. Health care providers' knowledge, attitudes, and self-efficacy for working with patients with spinal cord injury who have diverse sexual orientations. Phys Ther 2008; 88: 191-198.

12 Grabois EW, Nosek MA, Rossi CD. Accessibility of primary care physicians' offices for people with disabilities. An analysis of compliance with the Americans with Disabilities Act. Arch Fam Med 1999; 8: 44-51.

13 Schopp LH, Sanford TC, Hagglund KJ, Gay JW, Coatney MA. Removing service barriers for women with physical disabilities. Promoting accessibility in the gynecologic care setting. J Midwifery Women's Health 2002; 47: 74-79.

14 Fouts BS, Andersen E, Hagglund K. Disability and satisfaction with access to health care. J Epidemiol Community Health 2000; 54: 770-771.

15 Zhang J, Yuge Z, Genlin L, Yuchen G, Suwen C. Survey of reproduction needs and services: situation of persons with spinal cord injuries. Disabil CBR Inclusive Dev 2014; 25: 21-34.

16 de Groat WC, Kawatani M, Hisamitsu T, Cheng CL, Ma CP, Thor K et al. Mechanisms underlying the recovery of urinary bladder function following spinal cord injury. J Auton Nerv Syst 1990; 30: S71-S77.

17 Gerridzen RG, Thijssen AM, Dehoux E. Risk factors for upper tract deterioration in chronic spinal cord injury patients. J Urol 1992; 147: 416-418.

18 Biering-Sorensen F, Bagi P, Hoiby N. Urinary tract infections in patients with spinal cord lesions: treatment and prevention. Drugs 2001; 61: 1275-1287.

19 Kuo HC. Quality of life after active urological management of chronic spinal cord injury in eastern Taiwan. Eur Urol 1998; 34: 37-46.

20 Holroyd-Leduc JM, Straus SE. Management of urinary incontinence in women: scientific review. JAMA 2004; 291: 986-995.

21 Pannek J, Bertschy S. Mission impossible? Urological management of patients with spinal cord injury during pregnancy: a systematic review. Spinal Cord 2011; 49: 1028-1032.

22 Bertschy S, Geyh S, Pannek J, Meyer T. Perceived needs and experiences with healthcare services of women with spinal cord injury during pregnancy and childbirth - a qualitative content analysis of focus groups and individual interviews. BMC Health Serv Res 2015; 15: 234

23 Post MWM, Brinkhof MWG, von Elm E, Boldt C, Brach M, Muff C et al. Design of the Swiss Spinal Cord Injury Cohort Study (SwiSCl). Am J Phys Med Rehabil 2011; 90: 5-16.

24 Miles MB, Huberman AM. Focusing and Bounding the Collection of Data: The Substantive Start. 2nd edn. Sage: Thousand Oaks, CA, 1994.

25 Jensen MP, Truitt AR, Schomer KG, Yorkston KM, Baylor C, Molton IR. Frequency and age effects of secondary health conditions in individuals with spinal cord injury: a scoping review. Spinal Cord 2013; 51: 882-892.

26 Krassioukov A, Cragg JJ, West C, Voss C, Krassioukov-Enns D. The good, the bad and the ugly of catheterization practices among elite athletes with spinal cord injury: a global perspective. Spinal Cord 2015; 53: 78-82. 
27 Zimakoff J, Stickler DJ, Pontoppidan B, Larsen SO. Bladder management and urinary tract infections in Danish hospitals, nursing homes, and home care: a national prevalence study. Infect Control Hosp Epidemiol 1996; 17: 215-221.

28 Edwards JA, Reid YJ, Cozens DD. Reproductive toxicity studies with oxybutynin hydrochloride. Toxicology 1986; 40: 31-44.

29 Jia C, Liao LM, Chen G, Sui Y. Detrusor botulinum toxin A injection significantly decreased urinary tract infection in patients with traumatic spinal cord injury. Spinal Cord 2013; 51: 487-490.

30 Krasmik D, Krebs J, van Ophoven A, Pannek J. Urodynamic results, clinical efficacy, and complication rates of sacral intradural deafferentation and sacral anterior root stimulation in patients with neurogenic lower urinary tract dysfunction resulting from complete spinal cord injury. Neurourol Urodyn 2014; 33: 1202-1206.
31 Adriaansen JJ, van Asbeck FW, van Kuppevelt D, Snoek GJ, Post MW. Outcomes of neurogenic bowel management in individuals living with a spinal cord injury for at least 10 years. Arch Phys Med Rehabil 2015; 96: 905-912.

32 Ghidini A, Healey A, Andreani M, Simonson MR. Pregnancy and women with spinal cord injuries. Acta Obstet Gynecol Scand 2008; 87: 1006-1010.

33 Jackson AB, Wadley V. A multicenter study of women's self-reported reproductive health after spinal cord injury. Arch Phys Med Rehabil 1999; 80: 1420-1428.

34 Sterling L, Keunen J, Wigdor E, Sermer M, Maxwell C. Pregnancy outcomes in women with spinal cord lesions. J Obstet Gynaecol 2013; 35: 39-43.

35 Statistik BF. Europäischer Bericht zur Perinatalgesundheit 2010 Die Schweiz liegt bis auf wenige Ausnahmen im Durchschnitt. Neuchatel, 2013. 